\title{
Dual system increases the resolution of a low coherence interferometer system through a signal detector astigmatic focus
}

\section{Sistema dual que incrementa la resolución de un sistema interferométrico de baja coherencia por medio de un detector de señal de foco astigmático}

\author{
A. Eneas N. Morel, A. D. Aguilar, J. R. Torga \\ Universidad Tecnológica Nacional Facultad Regional Delta, Campana, Buenos Aires, Argentina. \\ ${ }^{(*)}$ E-mail: nmorel@frd.utn.edu.ar \\ Received: 04/06/2015 Accepted: 29/10/2015 \\ DOI: $10.7149 /$ OPA.48.4.301
}

\begin{abstract}
:
The low coherence interferometry in Fourier space (FD-OCT) is a technique to measure distance with typical values of dynamic range in the order of $3 \mathrm{~mm}$ and resolution below 10 microns. In this paper we propose to combine the interferometric technique with a technique that measures distances using astigmatic focus signal, allowing lead the resolution limit of less than $10 \mathrm{~nm}$.

The experimental scheme shown armed with both techniques and experimental results in displacement of a sample surface, controlled feedback system with a piezoelectric strain gauge which we have used as a reference system.

Key words: OCT, low coherence interferometry, focus signal, displacement measurement and tomography

\section{RESUMEN:}

La interferometría de baja coherencia en el espacio de Fourier (OCT-FD) es una técnica que permite medir distancia con valores típicos de rango dinámico en el orden de $3 \mathrm{~mm}$ y resolución por debajo de los $10 \mu \mathrm{m}$. En este trabajo se propone combinar esta técnica interferométrica con una técnica que mide distancias utilizando la señal de foco astigmática, lo que permite llevar el límite de resolución a menos de $10 \mathrm{~nm}$.

Se muestra el esquema experimental armado con ambas técnicas y resultados experimentales en desplazamientos de una superficie muestra, controlada con un sistema piezoeléctrico realimentado con strain-gauge que hemos utilizado como sistema de referencia.
\end{abstract}

Palabras clave: OCT, interferometría de baja coherencia, señal de foco, medición de desplazamientos y tomografías

\section{REFERENCES AND LINKS / REFERENCIAS Y ENLACES}

[1] I. Grulkowski, J.J. Liu, B. Potsaid, V. Jayaraman, J. Jiang, J.G. Fujimoto, A.E. Cable "High-precision, highaccuracy ultralong-range swept-source optical coherence tomography using vertical cavity surface emitting laser light source," Opt. Lett, 38, 5, 674-675 (2013); http://dx.doi.org/10.1364/0L.38.000673

[2] D. Huang, E. A. Swanson, C. P. Lin, J. S. Schuman, W. G.Stinson, W. Chang, M. R. Hee, T. Flotte, K. Gregory, C. A. Puliafito, J. G. Fujimoto, Science 254, 1178-1181 (1991). http://dx.doi.org/10.1126/science.1957169

[3] W. Drexler, J. Fujimoto, Editors, Optical Coherence Tomography: Technology and Applications, Springer (2008).

http://dx.doi.org/10.1007/978-3-540-77550-8 
[4] D. Stifter, K. Wiesauer, M. Wurm, E. Leiss, M. Pircher, E. Götzinger, B. Baumann, C. Hitzenberger, "Advanced optical coherence tomography techniques: novel and fast imaging tools for nondestructive testing," presented at The 17th World Conference on Nondestructive Testing, Shanghai, China, 25-28 October, (2008).

http://www.ndt.net/article/wcndt2008/papers/456.pdf

[5] C. During, S. Anderson and J. Wilkander, "Non-contact absolute measurement," Sensor Actuators A 32, 575-581 (1992).

http://dx.doi.org/10.1016/0924-4247(92)80046-6

[6] K. C. Fan, Y. T. Fei, X. F. Yu, Y. J. Chen, W. L. Wang, F. Chen, Y. S. Liu, "Development of a low-cost microCMM for 3D micro/nano measurement,” Meas. Sci. Technol. 17, 524-532, (2004). http://dx.doi.org/10.1088/0957-0233/17/3/S12

[7] C. Ruberti, E.N. Morel, J.R. Torga “Medición de distancias por señal de foco," Proceedings from Congreso Argentino de Ingeniería - CADI, Mar del Plata (2012).

[8] E.N. Morel, J.R. Torga “Limitaciones debido al detector en la interferometria de baja coherencia," Opt. Pura Apl. 41, 69-74 (2008)

[9] E. N. Morel, J. R. Torga "Simple method for thickness measurement in opaque samples with a Michelson-Sagnac interferometer," RIA0/OPTILAS: 6th Ibero-American Conference on Optics (RIAO); 9th Latin-American Meeting on Optics, Lasers and Applications (OPTILAS) Campinas, São Paulo, Brazil, AIP Conference Proceedings 992, pp. 793-797 (2007).

[10] E.N. Morel, M.V. Gutierrez, H.M. Miranda, E.L. Sambrano, J.R. Torga "Optical coherence tomographybased scanning system for shape determination, wall thickness mapping, and inner inspection of glass containers," Appl. Opt. 52, 1793-1798 (2013).

http://dx.doi.org/10.1364/A0.52.001793

\section{Introducción}

Las técnicas de interferometría óptica de baja coherencia han tenido en los últimos años un gran auge debido a que ofrecen la posibilidad de medir distancias, sin contacto y con alta resolución (inferior al micrón) [1-4]. Para el caso de muestras transparentes o semitransparentes (vidrios, polímeros, tejidos biológicos) estas técnicas ofrecen un atractivo adicional que es la posibilidad de realizar mediciones en el interior del material en lo que se conoce como tomografía óptica [2].

La Tomografía Óptica Coherente (OCT) se ha desarrollado notablemente en los últimos años en aplicaciones relacionadas con la medicina [3].

Recientemente ha tenido un gran impulso en el área de los ensayos no destructivos y estudios de materiales [4]. Esta técnica es utilizada normalmente en tres modalidades, en el dominio del tiempo (OCTTD), en el dominio de la frecuencia (OCT-FD) y utilizando fuentes de barrido (OCT-SSD). En aplicaciones donde el objetivo es obtener mediciones in situ y en tiempo real, es utilizada predominantemente la modalidad en el dominio de la frecuencia (FD) porque permite esquemas experimentales compactos y robustos, que pueden resolverse en sistemas integrados con fibra óptica y sin partes móviles. En una configuración típica se tiene una resolución espacial cercana a los 10 micrones y un rango de medición cercano a los $3 \mathrm{~mm}$.

En contraste con la interferometría de baja coherencia la técnica denominada por señal de foco astigmatica puede ser utilizada también para medición de distancias pero en su configuración típica ofrece una resolución espacial cercana al nanómetro y un rango de medición cercano a los 10 micrones [5-7].

El presente trabajo se centra en la utilización de un sistema que combine ambas técnicas de manera de tener un sistema con un rango dinámico para medición de distancias en el orden del mm con una resolución en el orden del $\mathrm{nm}$.

\section{Desarrollo}

Una medida típica de distancia hecha por interferometría de baja coherencia en el dominio de la frecuencia requiere la medición de la diferencia de camino óptico entre un punto medido sobre la muestra ( $\mathrm{x}_{1}$ en la Fig. 1) y un punto en la rama de referencia (usualmente un espejo, $\mathrm{x}_{2}$ en la Fig. 1). La medida se 
obtiene a partir de la frecuencia de modulación en el espectro de la fuente de luz, medida comúnmente con un espectrómetro.

El límite de resolución puede estar por debajo de la longitud de coherencia de la fuente (típicamente 10 $\mu \mathrm{m}$ ) y está limitado por varios factores como el ruido de la señal, la cantidad de píxeles y el número de bits del espectrómetro [2 y 8].

La técnica de señal de foco astigmatica [5 y 6] utiliza un esquema basado en el astigmatismo de la óptica de enfoque. En este caso el rango depende del sistema óptico utilizado, que para los sistemas comerciales se encuentra en el orden de los $10 \mu \mathrm{m}$. La resolución está definida por el ruido en el sistema de detección, generalmente un fotodetector de cuatro cuadrantes y su sistema de procesamiento analógico de señales. La salida de la etapa de procesamiento analógico se conecta a un sistema de adquisición de señales, este impone la resolución en función de la cantidad de bits que su conversor posee.

\section{2.a. Tomografía óptica en el dominio de la frecuencia.}

El sistema interferométrico utilizado en este trabajo se muestra en la Fig. 1. La fuente de luz es un diodo superluminiscente con una longitud de onda central de $840 \mathrm{~nm}$ y un ancho de banda de $60 \mathrm{~nm}$ con salida en fibra (Superlum 351 -HP3), que se conecta a un divisor de haz 50/50, las salidas del divisor se dirigen, una al espejo de referencia y la otra a la muestra, la luz reflejada en la muestra y en la referencia se recombinan generando la señal de interferencia, que se obtiene en la salida del divisor y que es detectada por un espectrómetro con un ancho espectral de 740 a 920 nm. (Ocean Optics HR-4000).

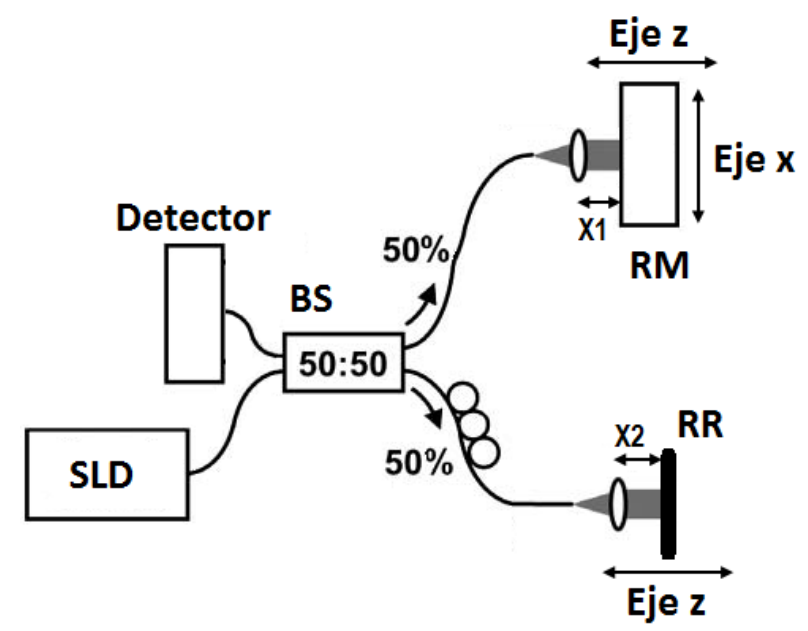

Fig. 1. Sistema interferométrico en fibra, SLD es el diodo superluminiscente, BS es el divisor de haz 50/50, RM es la rama de muestra y RR la rama de referencia.

Tanto el espejo de referencia como la muestra se encuentran montados en sendos sistemas de posicionamiento que permite trasladarlos a voluntad con fines de ajuste del interferómetro.

La medición de distancia se obtiene haciendo la diferencia $\Delta \mathrm{x}=\mathrm{x}_{2}-\mathrm{x}_{1}$.

\section{2.b. Sistema de señal de foco astigmático}

Este sistema se basa en un esquema experimental como se muestra en la Fig. 2. El diodo láser emite un haz linealmente polarizado que es difractado por una red de difracción. El orden central (orden 0) y los órdenes (1) y (-1) emergentes de la red inciden sobre un divisor de haz por polarización y sobre una lámina cuarto de onda. Luego, los haces son focalizados por una lente convergente sobre la muestra en estudio. El haz reflejado en la muestra pasa por la lámina cuarto de onda y por el divisor de haz y luego se dirige a los sensores. El orden 0 converge en un sensor 4 cuadrantes.

Llamando A, B, C y D a cada uno de los cuadrantes (fotodiodos) del sensor, se define la señal suma (SUM), a la intensidad total de luz, obtenida en los cuatro cuadrantes $(A+C+D+B)$ y la señal de error de foco (FE, $o$ señal de foco FC) definida como la resta de las intensidades medidas por los sensores opuestos $((A+D)-$ $(\mathrm{C}+\mathrm{B}))$.

El principio de medición de distancia se basa en el valor de la señal de foco obtenida en función de la posición de la superficie muestra con respecto al foco de la lente del cabezal, Fig. 2. 


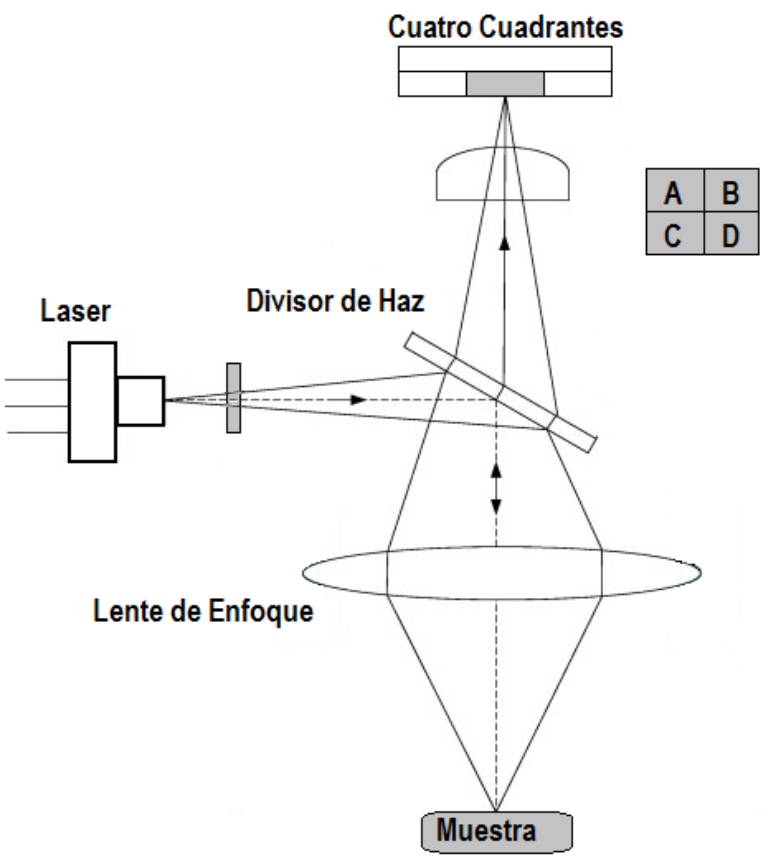

Fig. 2. Esquema simplificado del sistema astigmático.

Si la superficie se encuentra fuera del plano focal la forma del spot es la de una elipse, en ese caso la señal FE es distinta de cero y su módulo es creciente a medida que la distancia entre la muestra y el plano focal aumenta. En esta idea se basa el principio de medición que denominamos por señal de foco [7].

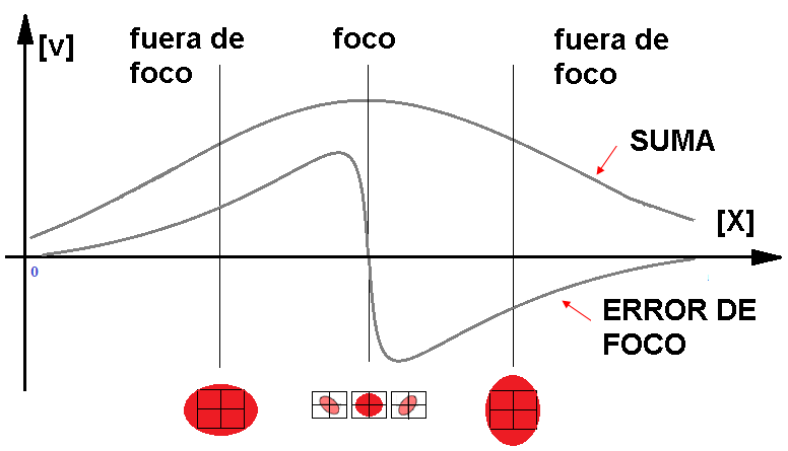

Fig. 3. Señales error de foco y suma.

Cuando la superficie muestra se encuentra en el plano focal de la lente la forma del spot del láser en el sensor es circular obteniéndose de esta manera un cero en la señal FE, cuando la muestra se desenfoca se genera una elipse que da más intensidad a los cuadrantes A y D o B y C según si la muestra se desplazó delante o detrás del plano focal, esta importante característica se logra gracias al astigmatismo y permite determinar distinguiblemente el avance o retroceso de la muestra, Fig. 3.

\section{Sistema compuesto}

En trabajos previos [8-10], se realizaron estudios en diversas aplicaciones utilizando OCT-FD en donde se pudo observar la problemática que se presenta al intentar medir variaciones por debajo de $1 \mu \mathrm{m}$. Esto se suma a las problemáticas vistas en el sistema astigmático debido a que su rango dinámico no supera los $10 \mu \mathrm{m}$.

Teniendo en cuenta las características de ambas técnicas, se decidió implementar un sistema compuesto que permita tener la versatilidad de ambas. Esto es un amplio rango dinámico del orden de los $3 \mathrm{~mm}$, con una resolución del orden de los nanómetros.

En la Fig. 4, se observa el esquema experimental del sistema compuesto donde se muestra el montaje del cabezal de señal de foco astigmático (CA) en la rama de muestra del interferómetro. 


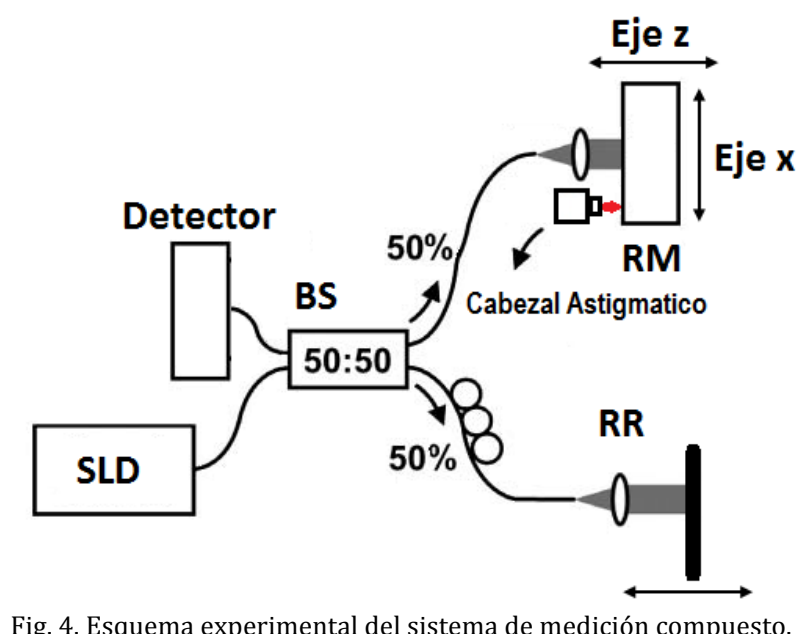

La muestra en estudio es un espejo de primera cara montada sobre un actuador piezoeléctrico.

Para realizar movimientos en escala de cientos de nanómetros de forma controlada que permitan llevar a cabo esta calibración se utilizaron actuadores piezoeléctricos. Los modelos utilizados son AE505D16F y PZS001 de la firma Thorlabs. El control de los actuadores se realizó mediante un controlador de la misma marca modelo TPZ001.

Los actuadores piezoeléctricos (PZ) tienen una respuesta no lineal y presentan histéresis, inconveniente que se debía salvar para poder posicionarnos correctamente. Para eso se incorporó al sistema el actuador PZS001 que viene instrumentado con un puente Wheatstone completo de galgas extensiométricas, un preamplificador y un lector de las galgas extensiométricas de la misma firma modelo TSG001 que en conjunto conforman un lazo cerrado de control, realimentado por el puente de galgas, el amplificador y el lector. El esquema completo se muestra en la Fig. 5. Al cerrar el lazo la respuesta del sistema se linealiza y se evita el problema de la histéresis. Con este sistema se logra un error de posicionamiento menor a $5 \mathrm{~nm}$.

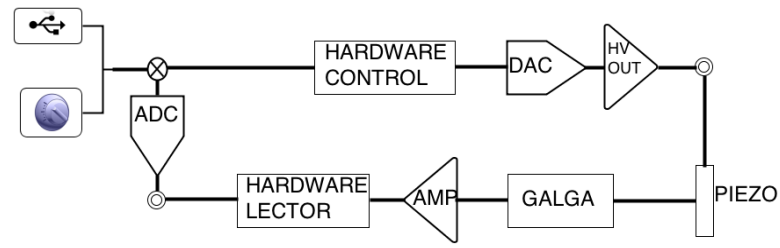

Fig. 5. Sistema de control del actuador piezoeléctrico

\section{Mediciones}

Es importante notar que ambos sistemas requieren una calibración previa [7-9]. En el caso de OCT-FD al ser la respuesta lineal se obtiene una constante de calibración mientras que el CA tiene una respuesta nolineal y requiere de una curva de calibración. Esta curva puede ser normalizada para independizar la técnica de la superficie a medir [6 y 8]. Cerca del foco la curva se puede aproximar con una recta. En este trabajo utilizaremos un rango de medición más amplio siendo entonces necesario obtener un polinomio de calibración.

Realizando un desplazamiento controlado del PZ desde su longitud de reposo hasta un desplazamiento de $9 \mu \mathrm{m}$, con un error en cada paso de $5 \mathrm{~nm}$, se obtuvo la curva de calibración para el sistema CA que se muestra en la Fig. 6.

Esta curva representa la distancia que se desplaza la muestra (desplazamiento) en función de la tensión medida con el detector de cuatro cuadrantes, correspondiente a la señal de foco. Cada punto de la curva se obtiene promediando 10.000 valores de tensión con una dispersión de $0.7 \mathrm{mV}$. 


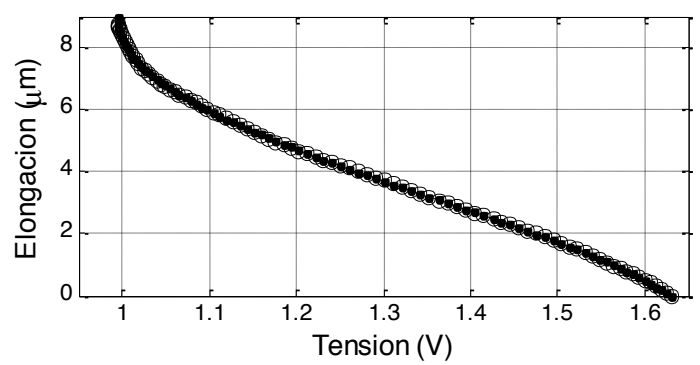

Fig. 6. Calibración Tensión vs. Desplazamiento, del CA.

La Fig. 6 muestra la curvatura presentes en sus extremos que evidencia que se utilizó el máximo rango de medición del sistema CA, partiendo de la cresta inferior a la cresta superior de la Fig. 3.

Un ajuste realizado con un polinomio de grado nueve, genera una curva con una desviación estándar con respecto a los datos originales de $52.66 \mathrm{~nm}$, cabe recordar que esta es la variación de la curva de ajuste y no el error de medición del CA.

Los valores de la dispersión de los datos medidos por el sistema CA, una vez afectados por la curva de calibración arrojan un valor promedio de $2.72 \mathrm{~nm}$.

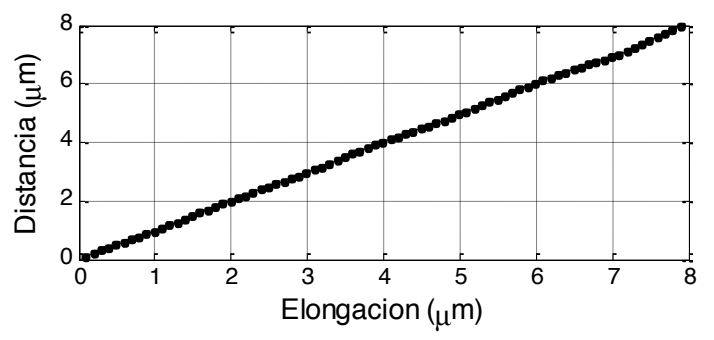

Fig. 7. Medida del desplazamiento vs distancia medida.

El sistema completo fue utilizado en una medición donde se fueron variando las distancias de desplazamiento de la muestra, utilizando el sistema PZ descripto anteriormente (Fig 5), en 19 pasos que se muestran en la Tabla 1.

TABLA 1. Valores de desplazamiento del PZ.

\begin{tabular}{|c|c|c|c|c|c|}
\hline \hline Pasos & $\begin{array}{c}\text { Distancias } \\
(\mu \mathrm{m})\end{array}$ & Pasos & $\begin{array}{c}\text { Distancias } \\
(\mu \mathrm{m})\end{array}$ & Pasos & $\begin{array}{c}\text { Distancias } \\
(\mu \mathrm{m})\end{array}$ \\
\hline \hline 1 & 0 & 7 & 0.3 & 13 & 0.001 \\
\hline 2 & 0.1 & 8 & 0.31 & 14 & 0.005 \\
\hline 3 & 0.2 & 9 & 0.315 & 15 & 0.01 \\
\hline 4 & 0.225 & 10 & 0.3155 & 16 & 0.02 \\
\hline 5 & 0.25 & 11 & 0 & 17 & 0.03 \\
\hline 6 & 0.275 & 12 & 0.0005 & 18 & 0.04 \\
\hline \hline
\end{tabular}

Los datos obtenidos se representan en la Fig. 8, en donde se comparan las medidas obtenidas con el sistema interferométrico (círculos), las medidas obtenidas con el puente de Wheatstone (diamantes) y las obtenidas con el sistema CA (cuadrados). 


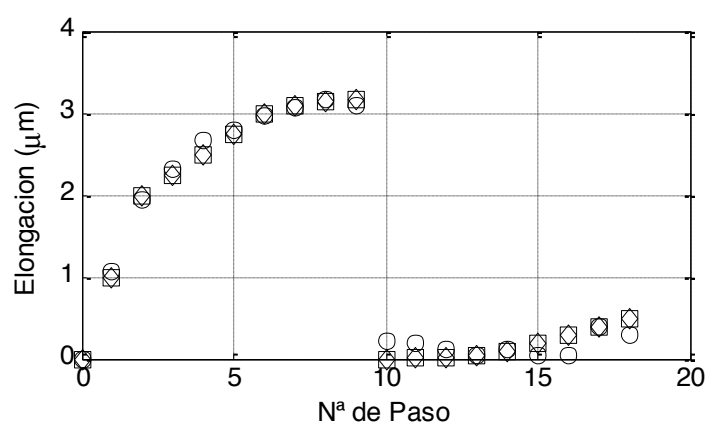

Fig. 8. En círculos medida tomada por el interferómetro, en diamante medida tomada por el puente de Wheatstone y en los cuadrados medida tomada por el sistema CA.

El error promedio de las medidas obtenidas con el interferómetro es de $0.5 \mu \mathrm{m}$. Esto es coincidente en orden de magnitud con el valor esperado $[3,4,10]$. En la Fig. 9 se muestra con mayor claridad los últimos puntos medidos (tabla 1), en donde se aprecia que el interferómetro posee un error mayor a los obtenidos con los sistemas CA y puente de Wheatstone.

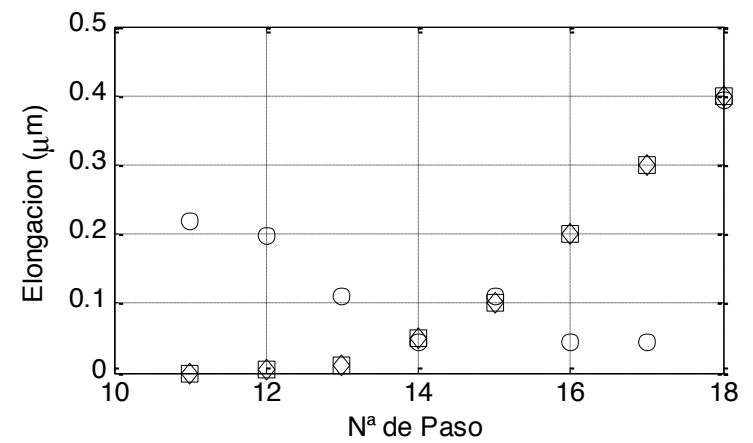

Fig. 9. Detalle ampliado de los últimos puntos medidos, mostrados en la figura 8 .

En este caso la dispersión en la medida del sistema CA es del orden de los $0.2 \mathrm{~nm}$, inferior a la obtenida con el puente de Wheatstone (sistema de referencia), por lo que se puede concluir que el error del sistema CA es inferior a los $5 \mathrm{~nm}$.

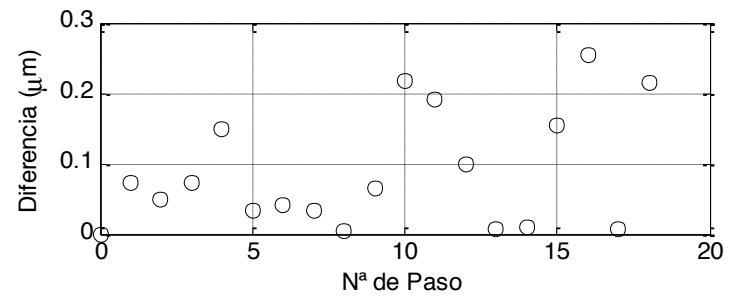

Fig. 10. Diferencia medida entre el sistema interferométrico y el sistema CA.

La diferencia entre las medidas obtenidas por el sistema interferométrico y el sistema CA, se pueden observar en la Fig. 10, donde se ve que coinciden en orden de magnitud con el error de medición del interferómetro.

\section{Conclusiones}

Se armó un equipo para medición de distancias combinando dos técnicas diferentes, que cubre un rango dinámico del orden de $3 \mathrm{~mm}$ con una precisión en el orden del nanómetro. La técnica de interferometría de baja coherencia provee el rango dinámico extenso, mientras que la señal de foco astigmática provee una alta resolución espacial (menor a $5 \mathrm{~nm}$ ).

Se encontró que el sistema CA debe posicionarse correctamente en el foco aprovechando al máximo su rango de medición. Claro está, que la respuesta del sistema CA presenta un comportamiento no lineal, por lo cual es necesario obtener una curva de calibración de calidad. Se debe notar que tanto el sistema 
interferométrico como el CA, son ambos vulnerables a las vibraciones mecánicas, exigiendo un ambiente de gran estabilidad.

Se logró combinar ambas técnicas mostrando que el agregado del cabezal CA permite mejorar la resolución espacial en por lo menos dos órdenes de magnitud, manteniendo el rango dinámico de medición del interferómetro.

\section{Agradecimientos}

Se desea agradecer la colaboración y financiamiento brindado por Universidad Tecnológica Nacional Facultad Regional Delta (FTN-FRD), Consejo Nacional de Investigaciones Científicas y Técnicas (CONICET). 